\title{
Certifying the interruption of Chagas disease transmission by native vectors: cui bono?
}

\author{
Fernando Abad-Franch ${ }^{1}{ }^{+}$, Liléia Diotaiuti ${ }^{2}$, Rodrigo Gurgel-Gonçalves ${ }^{3}$, Ricardo E Gürtler ${ }^{4}$ \\ ${ }^{1}$ Instituto Leônidas e Maria Deane-Fiocruz, Manaus, AM, Brasil ${ }^{2}$ Centro de Pesquisas René Rachou-Fiocruz, Belo Horizonte, MG, Brasil \\ ${ }^{3}$ Universidade de Brasília, Brasília, DF, Brasil ${ }^{4}$ Universidad de Buenos Aires, Buenos Aires, Argentina
}

Certifying the absence of Chagas disease transmission by native vectors lacks scientific grounds and weakens long-term control-surveillance systems to the detriment of people living under risk conditions. Instead, a regular "certification of good practice" (including vector control-surveillance, case detection/patient care and blood safety) could help achieve sustained disease control.

Key words: Chagas disease - vector control - interruption of transmission - certification

Chagas disease vector control is among the greatest public health achievements ever. The impact of the Inter-Government Initiatives coordinated by the Pan American Health Organization/World Health Organization (PAHO/WHO) has been enormous, with large reductions of incidence, prevalence and disease burden (Schofield et al. 2006). Yet, when entomological surveillance breaks down, native vectors re-invade and reinfest human residences, leading to the re-emergence of transmission (Gürtler et al. 2007). In fact, and as with other zoonoses, eradication of Chagas disease is impossible: sporadic Trypanosoma cruzi transmission to humans will always occur, even in the presence of the best conceivable control program (Schofield et al. 2006, Tarleton 2011).

Since the late 1990s, PAHO/WHO issue certifications of "interruption of T. cruzi transmission" by nonnative, accidentally introduced vectors. This approach was based on evidence that such vectors, and particularly Triatoma infestans and Rhodnius prolixus, were strictly domestic and did not occupy natural ecotopes in their non-native ranges. Therefore, it was argued, those introduced populations could be eliminated through insecticide-spraying campaigns. Empirical data show that this idea was basically right. T. cruzi transmission by domestic $T$. infestans populations was virtually or effectively interrupted in Uruguay (certified in 1997 and 2012), Chile (in 1999), Brazil (2006), eastern Paraguay (2008), some parts of Argentina (2001, 2004, 2011, 2012) and southern Peru and transmission by domestic $R$. prolixus populations in southern Mexico (2009), Guatemala

Financial support: FIOCRUZ, FAPEAM, CNPq, CAPES, CONICET + Corresponding author: fernando@amazonia.fiocruz.br

Received 11 December 2012

Accepted 23 January 2013
(2008), Honduras (2010), Nicaragua (2010), El Salvador (2010) and Costa Rica (2011) (Table I) (PAHO 2011, 2012, Hashimoto \& Schofield 2012).

Certification was intended to recognise the huge efforts made by Governments and international agencies to achieve the specific goal of interrupting transmission by these non-native vectors. Native vector populations, which occur in natural ecotopes across their geographic ranges, pose a radically different problem; here we argue that certification of interruption of transmission has no role to play in this case and question who is likely to benefit from such a certification policy.

We first wish to note that, on purely logical grounds, certifying the absence of an event is absurd - the absence of evidence cannot be taken as evidence of absence. In addition, native triatomines are nearly ubiquitous in the Americas; many species are very efficient vectors of $T$. cruzi and some are also capable on invading and colonising artificial environments (Lent \& Wygodzinsky 1979). Among these species are, of course, also T. infestans and $R$. prolixus, one native to the Chaco and the western Bolivian Andes and the other to the vast Orinoco plains (Table I). We also know, on empirical grounds, that sporadic vector-borne transmission does take place from the United States of America to Argentina, even in areas under tight surveillance - and we know that, unfortunately, most surveillance systems are far too weak to confidently rely on the data they produce (Abad-Franch et al. 2011).

What, then, would a "certification of interruption of transmission by native vectors" mean? Perhaps reflecting on a few recent examples (PAHO 2012) could help outline an answer. For what does it mean that T. cruzi transmission has been interrupted in, say, the Bolivian Department of La Paz or some localities of the Chaco in Argentina - where native T. infestans populations are common, surveillance is weak and most rural houses are still substandard? And what would it mean that transmission by native vectors no longer takes place in non-Amazonian Brazil - where over 50 triatomine species occur, a dozen of which are routinely collected inside houses? 
TABLE I

Current major scenarios and general strategies in relation to Chagas disease vector control-surveillance in Latin America

Scenario Vectors Countries Strategy

Areas where transmission by introduced domestic vectors has been interrupted

Areas where transmission by introduced domestic vectors has not yet been interrupted

Areas where major domestic vectors are native domestic vectors are native

Areas with potentially important domestic vectors

Areas where continuous transmission by nondomiciliated vectors has been documented (including oral transmission)

Areas where sporadic transmission by nondomiciliated vectors has been documented (including oral transmission)
Rhodnius prolixus

Triatoma dimidiata

R. prolixus

T. infestans

T. dimidiata

R. prolixus

T. infestans

T. dimidiata $\mathrm{s} .1$.

Mainly $T$. dimidiata s.1., Triatoma pallidipennis, Triatoma barberi, Rhodnius ecuadoriensis, Triatoma carrioni, Triatoma maculata, Panstrongylus megistus, Triatoma brasiliensis s.1., Triatoma pseudomaculata

Several phyllosoma group species, Triatoma venosa,

Panstrongylus chinai,

Panstrongylus howardi, Panstrongylus herreri,

Rhodnius stali, Triatoma

sordida, Panstrongylus rufotuberculatus,

Panstrongylus lutzi,

Rhodnius neglectus,

Rhodnius nasutus, Triatoma rubrovaria, among others

Mainly Rhodnius pallescens, Rhodnius robustus s.l., Rhodnius pictipes, Rhodnius brethesi, Panstrongylus geniculatus

Many species
S Mexico ${ }^{a}$, Guatemala $^{a}$, Honduras $^{a}$, El Salvador ${ }^{a}$, Nicaragua ${ }^{a}$, Costa Rica $^{a}$

Uruguay $^{a}$, Chile $^{a}$, Brazil $^{a}$, parts of Argentina $^{a}$, E Paraguay $^{a}$

NW Peru

NW Colombia

S Peru

W Ecuador

Colombia, N Venezuela

Bolivia, W Paraguay, N Argentina, central-N Chile

Colombia, N Venezuela, Mexico, all Central American countries

Mexico, all Central American countries, Colombia, Venezuela, Brazil, Ecuador, Peru, Bolivia,

Paraguay, Argentina, Chile
Stringent surveillance to detect and eliminate infestation foci, whether residual or resulting from re-introduction

Area-wide surveillance and control

Area-wide surveillance with focal control, particularly in rural and periurban settings

Area-wide surveillance with focal control, particularly in rural and periurban settings
Mexico, all Central American countries, Colombia, Venezuela, Brazil, Ecuador, Peru, Bolivia, Uruguay, Paraguay, Argentina, Chile
Area-wide surveillance with focal control, particularly in rural and periurban settings

$a$ : certified countries (or parts thereof) [note that the Bolivian Department of La Paz and the southern Peruvian Departments of Tacna and Moquegua have also been certified and that several further certification assessments are underway (PAHO 2012)].We stress that these strategies must always be accompanied by (i) serological screening of all blood and organ donors, (ii) integral patient care (diagnosis, specific chemotherapy and palliative treatment, follow-up and general support) at the primary health care level, with emphasis on reproductive-age women and with an adequately designed referral system for more severe cases and (iii) operational research (e.g., environmental management, physical protection of houses and food-processing premises, improved vector-detection systems or monitoring of insecticide resistance). 
One hardly controversial fact is that all available entomological data are extremely fragmentary and become even more so as funds decline. Furthermore, we must remember that acute Chagas disease is "typically" asymptomatic or oligosymptomatic (Rassi et al. 2010) and goes often undiagnosed, and that vector detection methods all have very low sensitivity (Abad-Franch et al. 2011). Will the absence of detection be interpreted as true absence of events? (and would a single new case result in "de-certification"?). Note also that even the best possible serological survey of children born in the "post-control era" would not be truly informative about the occurrence of "sporadic" transmission. The last large survey was conducted in Brazil about seven years ago and it seems unlikely that many of similar size (and costs) will be conducted in the future, because no political priority can be attached to a disease whose transmission by its main domestic vectors has been (officially) "interrupted". Would a decision-maker devote funds to monitoring the possible existence of events that have been declared inexistent by the most authoritative international health agency? Again, we think this highly unlikely; on the contrary, "certification" has usually had conspicuous "fund-freezing", priority-lessening effects. This, of course, weakens surveillance and a weaker surveillance system produces fewer records regardless of epidemiological reality, reinforcing the impression that transmission does not occur. Such a positive feed-back loop generates a false feeling of safety, with transmission remaining overlooked until the pre-control situation is restored - and control investment wasted for good. This has been quite rightly called "the punishment of success" (Schofield et al. 2006), a phenomenon whose grave effects are, unfortunately, well documented (Gürtler et al. 2007).

Should then PAHO/WHO stop certifying altogether? We do not think so: publicly acknowledging the efforts and achievements of health authorities is both fair and important. But we need to ask and answer the question we chose for our title: cui bono - who benefits from certification? As it currently stands, certification effectively discourages sustained action rather than promoting it and this surely does not benefit people living under risk conditions: they deserve better. Perhaps the simplest strategy would be to certify "good practice" instead of unverifiable facts about the non-occurrence of transmission. This would have several advantages. First, "good practice" is a dynamic concept - it does not focus on a "state" to be reached, but on better ways of running long-term programs, encouraging decision-makers to improve performance and secure adequate funds. Second, "good practice" can be assessed periodically based on quantitative indicators (Table II); in defining these indicators, issues of validity, reliability, comparability and ease of ascertainment must be taken into account (Murray 2007). Third and perhaps most important, "good practice" can be defined as encompassing the several key aspects of Chagas disease control, including not only vector control-surveillance, but also case detection/patient care and blood safety - it might even include a technical assessment of financial management. Thus, "good practice" evaluations can generate a more comprehensive picture of how control programs are performing, providing the means to dynamically detect and correct malfunctions. In the long run, "good prac-

TABLE II

Sustained Chagas disease vector control and surveillance: key points for functioning and assessment

What must be done

Every basic administrative unit in charge of vector-borne disease control-surveillance must have a written plan of activities related to Chagas disease. Such plans must:

follow national/regional technical guidelines;

establish clear goals and timelines;

have adequate funds;

provide technicians with the continuous training, equipment and materials needed to (i) carry out fieldwork, (ii) apply insecticides, (iii) identify vectors and parasites in the laboratory and (iv) regularly fill data forms and submit them to regional/national registries.

Every secondary administrative unit (from state/province/department to country) must have a supervision team capable of providing technical support to regional/local teams (including the production and distribution of guidelines) and periodically collecting, managing and synthesizing the data those teams produce.

What should, in addition, be done

Chagas disease control-surveillance plans should incorporate community participation in vector detection and reporting; a strong component of health education, information and communication is required to stimulate participation.

Local plans should be adapted to the ecological and social reality; regional/national technical guidelines should be flexible enough to allow for such adaptation.

Operational research should become a routine tool to improve surveillance; local, regional and/or national health authorities should actively seek support from academic researchers to design projects aimed at answering practical, high-priority questions. 
tice" certification could be crucial to the strengthening of control programs and the prevention of new Chagas disease cases. It would thus directly benefit those at risk, whereas certifying interruption of transmission, we suspect, primarily benefits authorities.

In conclusion, we strongly advocate the idea that $\mathrm{PAHO} / \mathrm{WHO}$, the relevant public health officials and the scientific community should engage in a serious debate to define better ways to monitor the performance of Chagas disease control programs. Certifying interruption of transmission has many conceptual drawbacks and effectively weakens long-term control-surveillance systems; in contrast, periodically assessing "good practice" would stimulate sustained action and could therefore represent a major step in the right direction. It may well be, in fact, the best way forward.

\section{ACKNOWLEDGEMENTS}

To the memory of Antônio Carlos Silveira.

\section{REFERENCES}

Abad-Franch F, Vega MC, Rolón MS, Santos WS, Rojas de Arias A 2011. Community participation in Chagas disease vector surveillance: systematic review. PLoS Negl Trop Dis 5: e1207.

Gürtler RE, Kitron U, Cécere MC, Segura EL, Cohen JE 2007. Sustainable vector control and management of Chagas disease in the Gran Chaco, Argentina. Proc Natl Acad Sci USA 104: 16194-16199.
Hashimoto K, Schofield CJ 2012. Elimination of Rhodnius prolixus in Central America. Parasit Vectors 5: 45.

Lent H, Wygodzinsky P 1979. Revision of the Triatominae (Hemiptera, Reduviidae) and their significance as vectors of Chagas disease. Bull Am Mus Nat Hist 163: 123-520.

Murray CJL 2007. Towards good practice for health statistics: lessons from the Millennium Development Goal health indicators. Lancet 369: 862-873.

PAHO - Pan American Health Organization 2011. Enfermedad de Chagas: transmisión por el principal vector. Available from: new. paho.org/ hq/images/stories/AD/HSD/CD/Chagas/int_trans_vector_chagas_800.jpg.

PAHO - Pan American Health Organization 2012. Reunión Sudamericana de Iniciativa Subregionales de Prevención, Control y Atención de la Enfermedad de Chagas, Buenos Aires, Argentina, 27-29 August 2012. Available from: new.paho.org/hq/index. php?option $=$ com_docman\&task $=$ doc_download\&gid $=18851 \&$ It emid $=270 \&$ lang $=$ en.

Rassi Jr A, Rassi A, Marin-Neto JA 2010. Chagas disease. Lancet 375: 1388-1402.

Schofield CJ, Jannin J, Salvatella R 2006. The future of Chagas disease control. Trends Parasitol 22: 583-588.

Tarleton RL 2011. Chagas disease impact and opportunities: beyond the historical dogma. Institute of Medicine (US) Forum on Microbial Threats. The causes and impacts of neglected tropical and zoonotic diseases: opportunities for integrated intervention strategies. Available from: ncbi.nlm.nih.gov/books/NBK62523/. 